\title{
Tecnología como factor de innovación en empresas colombianas
}

\author{
Londoño Rua, John Edisson
}

\section{Resumen}

El desarrollo de innovaciones depende de múltiples factores para lograr consolidar procesos exitosos que parten de la definición de una estrategia adecuada. Uno de los factores importantes es la tecnología dado que contribuye al logro de los resultados empresariales propuestos, por tanto, el objetivo del presente trabajo consiste en verificar si la adquisición de tecnologías de información genera beneficios en innovación para las empresas colombianas; así mismo, se analiza su importancia como insumo para la definición de la estrategia organizacional. Para ello se hizo un ejercicio de correlación estadística entre las variables de adquisición de tecnología e Innovación con elementos de la II Encuesta de Desarrollo e Innovación Tecnológica en Colombia, indicando si existe dependencia entre resultados de las variables. Como principal conclusión se tiene que en Colombia la adquisición de tecnología no es parte activa de la estrategia organizacional, o su uso se ha orientado hacia actividades que no generan ventaja competitiva. Mediante este estudio se pretende contribuir al desarrollo del proceso de gestión en las empresas del país.

Palabras clave: Innovación; tecnología; estrategia de innovación; empresas colombianas.

\section{Technology as an innovation factor in Colombian enterprises}

\begin{abstract}
The development of innovations depends on multiple factor in order to achieve consolidation of successful projects which come from the definition of an adequate strategy. One of the factors is technology given that it contributes to the achievement of the proposed business results, therefore, the aim of the present study consist of verifying if the acquisition of information technology generates innovation benefits for the Colombian enterprises; furthermore, analyzes its importance as an input to the definition of organizational strategy. In order to do so, a statistic correlation exercise between the variables of technology an innovation acquisition and elements of the II Development and Technological Innovation Survey in Colombia was made, indicating if dependence between the variables results exists. As a main conclusion, the technology acquisition in Colombia is not
\end{abstract}

Recibido: 08.12.14. Aceptado: 12.04.15

* Candidato a Doctor en Ingeniería - Magíster en Ingeniería Administrativa - Profesor Tiempo Completo del Instituto Tecnológico Metropolitano de Medellín. Email: johnlondono@itm.edu.co 
an active part of the organizational strategy or its use has been oriented to activities which do not generate competitive advantage. This study is intended to contribute to the management process development in the companies of the country.

Key Words: Innovation; technology; innovation strategy; Colombian enterprises.

\section{Introducción}

La tecnología afecta a las organizaciones de diversas formas; siendo un factor clave la determinación delas tareasyel grado de especialización requeridos (Kast y Rosenzweig, 1988). Es así como la relevancia del papel de la tecnología es evidente tanto en el nivel productivo como en la gestión global, y en el caso de las tecnologías de avanzada (nanotecnología, biotecnología, electrónica y tecnologías de información y comunicaciones TIC), contiene un impacto directo y profundo, en gran cantidad de sectores y empresas. Sin embargo, lo que no se observa fácilmente es si el impacto está dado por el mero hecho de obtener la tecnología y operar en otras condiciones.

Por ende, este trabajo consiste en verificar si la adquisición de un tipo de tecnología específica, como la TIC, está relacionada con la innovación, como un resultado específico beneficioso en las empresas colombianas, producto de la definición de estrategias organizacionales. Sin embargo, se debe tener en cuenta que "no es posible generalizar que la alta tecnología juega un papel importante en el desarrollo de la innovación en todos los sectores y empresas en el ámbito Colombiano, por tanto se debe promover la creación de políticas para dinamizar el uso de estas tecnologías en las empresas" (Londoño, 2010:61).
Para lograr este objetivo se hizo una exploración de la literatura disponible en bases de datos científicas, complementada con un análisis estadístico a partir de la información de la segunda Encuesta de Desarrollo e Innovación Tecnológica (EDIT II) realizada por el Departamento Administrativo Nacional de Estadística -DANE- en Colombia. Para ello se utilizaron dos variables: 1) la Adquisición de Tecnologías y, 2) la innovación de productos, para las cuales se verificó la existencia de la relación y la intensidad de la misma.

Para el análisis, se hace énfasis en la noción de actividad de innovación del Manual de Oslo, específicamente en la posibilidad que una empresa que desarrolle este tipo de actividades como tal no produzca resultados en innovación.

\section{Tecnología e Innovación en las organizaciones}

Para comprender el alcance de la tecnología en las organizaciones es necesario hacer referencia a la definición del término, para no confundirla con los productos que se derivan del uso o aplicación de esta. Además es importante tener en cuenta que en este trabajo, se analiza el concepto de tecnología a nivel organizacional. Para definir el concepto de tecnología se presentan diferentes enfoques que permiten ilustrar mejor el alcance de la misma para las organizaciones. 
Para O'Regan y Sims (2008), existen muchas definiciones de tecnología, pero resaltan entre las más importantes dos orientadas a la transformación de materias primas en bienes y servicios. Rosenberg (1982) y Galbraith (1967) se enfocan en la aplicación sistemática del conocimiento científico, por tanto la importancia de diferenciar técnica y tecnología, aunque en su trabajo considera la técnica como parte de la tecnología. Por último, para Fernandez (2005) la tecnología cumple con determinadas características que la convierten en un bien y no es de uso libre, en donde hay que valorar la compatibilidad del sistema tecnológico con el entorno donde la empresa desarrolla su actividad. Adicionalmente, la tecnología va ligada a unas características que se deben considerar al momento de incorporarlas en la empresa, las cuales están planteadas por Fernandez (2005):

- Oportunidad: referida a las motivaciones que la tecnología puede ofrecer relacionadas con satisfacer o crear alguna necesidad del mercado y a la factibilidad con que pueden alcanzarse nuevos avances tecnológicos.

- Especificidad: posee un valor en su uso, superior al que poseería bajo cualquier otro usuario o uso alternativo. Lo es en dos sentidos: 1) respecto al entorno en donde se lleva a cabo la actividad tecnológica, y 2) respecto a los productos y procesos.

- Complejidad: en general, las tecnologías están formadas por múltiples componentes e interacciones y una parte de sus conocimientos es de naturaleza tácita. Todo ello hace compleja la tecnología, lo que dificulta su comprensión y asimilación.

- Acumulabilidad: significa que la actividad tecnológica en las empresas tiende a construirse gradualmente a partir de lo que ya se conoce, incluso cuando lo que se busca son grandes cambios o revoluciones.

- Apropiabilidad: término relacionado con la capacidad del propietario de la tecnología para capturar el valor derivado de la aplicación comercial de la misma.

En el enfoque organizacional, la tecnología se concibe como la aplicación sistémica del conocimiento por medio del cual se generan bienes y servicios disponibles para quien los requiera. Convirtiéndola en la principal "herramienta" con que las organizaciones cuentan para el diseño, creación, y distribución de sus productos y generando un carácter estratégico a la utilización y dominio de la misma.

Tanto en la gestión estratégica, como en la operativa, la tecnología se ha convertido en la mano derecha, junto con la administración, en el momento de trabajar por el desarrollo de los objetivos y metas de las organizaciones (Aït-El-Hadj, 1990). Se convierte entonces en un recurso fundamental para la obtención de ventajas competitivas y en la acumulación de capacidades de innovación.

Siguiendo a Eisenhardt y Martin (2000) el enfoque basado en los recursos de la empresa (RBV) es un marco influyente teórico para entender cómo se logra una ventaja competitiva en las empresas y cómo esa ventaja podría ser sostenida en el tiempo, por ende desde el enfoque basado 
en recursos es posible analizar la tecnología como un recurso estratégico para las organizaciones.

La literatura (Nelson 1991; Penrose 1959; Prahalad y Hamel 1990; Teece, Pisano, y Shuen 1997) ve los recursos (activos, capacidades, procesos organizacionales, información, conocimiento, entre otros.) (Barney, 1991:101) como el primer escalón en la cadena de valor y el conductor de las capacidades y posteriormente de la ventaja competitiva, esto indica que los recursos se necesitan para identificar, nutrir, desplegar y explotar la empresa para maximizar las oportunidades inherentes a la operación en el ambiente externo (O'Reagan y Sims; 2008)

Otra forma de recursos son las capacidades y competencias básicas de la organización con el fin de proveer beneficios a los clientes, convirtiéndose en un modelo a seguir para la competencia y así maximizar los beneficios resultantes de los productos o procesos obtenidos (O'Reagan y Sims; 2008). La relación entre el conocimiento y desempeño tienen una fuerte sustentación empírica que se centra en la capacidad de la empresa para crear, utilizar y desarrollar sus conocimientos basados en los activos; el conocimiento es considerado como uno de los principales impulsores de las capacidades

Robledo et al, (2009) plantea desde la perspectiva de los recursos y capacidades que detrás de la innovación como proceso están las capacidades organizacionales que la hacen posible. Ante la necesidad de gestionar adecuadamente esas capacidades y darles una aplicación estratégica en la organización, el papel de la gestión tecnológica es fundamental, al buscar la integración de la tecnología y la estrategia, así como la generación, difusión y aplicación de innovaciones. En este sentido, se busca incrementar las capacidades de la organización para explotar la tecnología disponible de manera que su uso tenga un impacto en diferentes ámbitos, y sea un soporte efectivo en la generación de innovaciones, que dependerá en gran medida de la manera como se utilice y aproveche el factor tecnológico y de las capacidades generadas a partir de ello; por tanto, la relación tecnología-innovación está mediada por la gestión organizacional.

El análisis de las capacidades y el papel de la gestión tecnológica en el logro de resultados innovadores ha sido abordado desde hace varias décadas, a partir de ópticas y contextos diversos, e involucrando conceptos como capacidades de innovación (Christensen, 1995; Burgelman et al, 2001) y competencias tecnológicas (Bell y Pavitt, 1995; Lall, 1992; Tidd, 2000), que buscan identificar las posibilidades para producir innovaciones como resultado de la gestión estratégica u operativa de la organización (Robledo et al, 2009). Las capacidades son las habilidades, experiencia y aptitudes que la organización logra desarrollar, por ende es posible afirmar que el producto de las capacidades de innovación y las competencias tecnológicas se materializa en las estrategias que la organización logra definir.

Hamel (1998) define la estrategia de innovación como la posibilidad de reciclarse en los distintos modelos de industria y a partir de los cuales se crea valor para los clientes, se ubica al frente de tus competidores, 
con nuevos productos y aportando riqueza para los accionistas. Markides (1997) por su parte, la define como una reconceptualización fundamental que se da cuando las organizaciones son capaces de reinventar la forma de jugar en el tablero empresarial. Para Freeman y Soete (1997) se centran en la definición de la mezcla productoproceso-mercado, que se puede simplificar en tecnología - Mercado.

De acuerdo a lo anterior, una estrategia de innovación son los compromisos o acciones específicas que en materia de innovación asume la organización, quien es representada por el órgano ejecutivo o quien haga sus veces, con el fin de otorgar valor a sus clientes y generar ventajas competitivas sostenibles en el tiempo.

\section{Análisis de la innovación}

El análisis de la innovación parte de las aportaciones de Schumpeter "quien introduce, como elemento central del cuerpo teórico del análisis económico, el proceso de innovación empresarial. Con su descomunal aportación al campo de estudio de este proceso y del cambio tecnológico, trasciende el pensamiento estático y de corto plazo que dominaba su época" (Olaya, 2008:239). Es así como Schumpeter (1967:76); citado por Olaya (2008:240), entendía la innovación como; "producir otras cosas, o las mismas por métodos distintos" Este concepto se concentra en el desarrollo y creación de nuevos bienes y el cambio en los métodos de producción, como elemento central para explicar el proceso.

La Organización para la Cooperación y Desarrollo Económicos -OCDE(2005:46) plantea una concepción de la innovación que va más allá del cambio tecnológico, definiéndola como "la introducción de un nuevo, o significativamente mejorado, producto (bien o servicio), de un proceso, de un nuevo método de comercialización o de un nuevo método organizativo, en las prácticas internas de la empresa, la organización del lugar de trabajo o las relaciones exteriores", en un sentido más general, se puede considerar según el americano Sherman Gee (1981), citado por Escorsa y Valls (1998:20) como: "el proceso en el cual a partir de una idea, invención o reconocimiento de una necesidad se desarrolla un producto, técnica o servicio útil hasta que sea comercialmente aceptado".

De acuerdo con esto, innovar es transformar un producto, servicio o forma de realizar una gestión de acuerdo con unas necesidades identificadas en el mercado, lo que traerá como consecuencia el logro de un objetivo económico.

Partiendo de la base que la adquisición de tecnologías es considerada por el Manual de Oslo como una actividad innovadora, es necesario tener en cuenta que el mero hecho de usar o adquirir Tecnologías de Información y Comunicaciones no representa como tal una innovación; a esto hace referencia el Manual cuando plantea: "es posible que haya quienes desarrollen actividades de innovación durante el período analizado en una encuesta sin haber introducido efectivamente una" (OCDE, 2005:71). En este trabajo se pretende verificar si existe una relación entre dicha adquisición con la introducción de innovaciones.

La innovación es generadora de rentabilidad y de incremento en los niveles de utilidad (Cory, 1999) y por 
ende hace parte fundamental en el proceso de gestión de la organización que le apunta a la diferenciación, además a largo plazo se convierte en un elemento estratégico al permitir aumentos en la cuota del mercado, en el posicionamiento, márgenes de utilidad y aprendizaje (Malaver, 2000). La innovación es importante entonces, porque se convierte en el eje fundamental de la competitividad a gran escala; aunque se puede ser competitivo manteniendo solo procesos de mejora continua, pero generalmente esto no es suficiente si se toma en cuenta, que existen necesidades en los clientes, que los productos o servicios existentes no logran solventar.

Es importante analizar el proceso a partir de las ideas que se llevan a un uso productivo contemplando dos fases: una creativa, donde se lleva a cabo la generación de la idea, y otra ejecutiva, donde esta se transforma en resultado (Snarch, 1998). Con el fin de orientar los procesos generales de la empresa, la orientación enmarca una gran oportunidad de ventaja competitiva para la misma, sin embargo, es necesario que se destinen una serie de recursos para llevar adelante este proceso y lograr obtener esas ventajas competitivas. Este trabajo plantea verificar ese uso de recursos mediante la hipótesis de que la adquisición de TIC está relacionada con la innovación.

\section{La estrategia de innovación}

Ante la necesidad de gestionar adecuadamente esas capacidades y los recursos tecnológicos y la necesidad de darles una aplicación estratégica, aparece el concepto de gestión tecnológica, que inicialmente hacía referencia a la administración de la investigación y desarrollo, pero que con el tiempo evolucionó y se enfocó en la planeación, la innovación y finalmente gestión de la tecnología como parte estratégica de la administración (Drejer, 1996).

Según Gallego (2005) la gestión tecnológica se define como el conjunto de decisiones vinculadas a la creación y/o adquisición, desarrollo y/o transformación y comercialización de la tecnología, desde un punto de vista estratégico como operacional que se materializa en el plan estratégico de desarrollo tecnológico.

La gestión tecnológica estratégica, está dada entonces por la integración de la tecnología y la estrategia como un enfoque plenamente integrador, para la administración que desconoce y explica el papel central de la tecnología y el desempeño de una firma, con una visión asociada de tecnología como soporte primario para productos y procesos mejorados (Cortés, 2004). A su vez, siguiendo a Morcillo (1997:107-108) citado por Gallego (2005), la gestión tecnológica se orienta también hacia la difusión y aplicación de la innovación.

Ahora también es posible identificar cómo los procesos de gestión tecnológica se convierten en una fuente estratégica de desarrollo que potencializa la generación de innovaciones. Uno de los conceptos que puede explicar esta aseveración es el de elasticidad de demanda tecnológica, es decir, la influencia sobre la demanda de un producto que tiene la mejora de uno o varios de sus parámetros (costo, consumo, duración, fiabilidad. entre otros.) (Roberts, 1995 citado por Escorsa y Valls, 1998); 
por ende, la utilización de procesos de gestión tecnológica permite a la organización establecer un factor de ventaja competitiva con el fin de obtener una posición defendible dentro de una industria (Porter, 1980).

La innovación representa una respuesta a las situaciones de cambio imprevisibles y se relaciona con los procesos globales de la organización, señalando una estrecha integración entre la tecnología y la gestión. Sus objetivos están centrados en neutralizar las amenazas creadas por la turbulencia tecnológica, apropiándose de todas las oportunidades que se puedan generar, además trata de potencializar los niveles de diferenciación que posee la empresa, así como su flexibilidad, agilidad y niveles de adaptación (AïtEl-Hadj, 1990).

En síntesis, para analizar el concepto de estrategia de innovación es posible abordar dos enfoques mediante los cuales se visualiza la tecnología y la innovación como factores estratégicos para la organización. En el primer enfoque, desde el análisis de la Gestión Tecnológica se llega a la estrategia, desde el planteamiento evolutivo por escuelas que hace Drejer (1996). En el segundo enfoque, se evidencia como desde la estrategia organizacional se identifica la necesidad de un área que gestione la tecnología para apoyar el desarrollo de la ventaja competitiva, como se puede identificar en Porter (1980) y Aït-El-Hadj (1990).

Sanches y Machado (2014) hacen una análisis de la estrategia de innovación a partir del Enfoque Basado en Recursos (RBV, por sus siglas en ingles), en donde se concluye que desde la perspectiva del modelo de recursos VRIO (recursos Valiosos, Raros, Inimitables e Inmersos en la Organización), los recursos intangibles se destacan, como es el caso del know-how, la cultura organizacional, la capacidad emprendedora y la capacidad de innovación.

Se concluye entonces que la estrategia de innovación esta permeada por la gestión que se hace sobre una serie de recursos, entre los cuales la tecnología juega un papel importante, que requiere de un proceso exclusivo de gestión, en busca del desarrollo de la ventaja competitiva y de la innovación como tal.

\section{Importancia de la tecnología en la estrategia de innovación}

Freeman (1982) busca categorizar las posibilidades estratégicas de la empresa en innovación, partiendo del análisis de la competencia en el mercado y la jerarquía en la que de acuerdo a las capacidades existentes puede enfocarse. Este análisis parte de la mezcla Proceso - Producto Mercado, que se este en capacidad de configurar y que puede simplificarse en Tecnología - Mercado, para ello propone la siguiente clasificación de estrategias (Cuadro 1). 


\section{Cuadro 1}

\section{Clasificación de Estrategia según Freeman}

\begin{tabular}{|c|c|}
\hline $\begin{array}{l}\text { TIPO DE } \\
\text { ESTRATEGIA }\end{array}$ & EXPLICACIÓN \\
\hline OFENSIVA & $\begin{array}{l}\text { Una estrategia de innovación ofensiva es la diseñada para lograr el liderazgo técnico y } \\
\text { de mercado, para estar todo el tiempo adelante de los competidores en la introducción } \\
\text { de nuevos productos". }\end{array}$ \\
\hline DEFENSIVA & $\begin{array}{l}\text { Quienes que siguen estrategias defensivas deciden no ser las primeras en llegar al } \\
\text { mercado con sus innovaciones y de esta manera buscan disminuir los riesgos y despejar } \\
\text { muchas incertidumbres relativas a la reacción del mercado ante la innovación, así como } \\
\text { aprovecharse de los errores que cometen los innovadores líderes. }\end{array}$ \\
\hline IMITATIVA & $\begin{array}{l}\text { Las organizaciones imitativas se caracterizan por introducir innovaciones con un retraso } \\
\text { significativo frente a los líderes. Ello sucede cuando las patentes ya están vencidas y las } \\
\text { tecnologías son de dominio público y de relativo fácil acceso. }\end{array}$ \\
\hline DEPENDIENTE & $\begin{array}{l}\text { Quienes siguen tales estrategias son fuertemente dependientes de la iniciativa de otras } \\
\text { empresas, usualmente más grandes y desarrolladas tecnológicamente, a las cuales } \\
\text { sirven como proveedores, por ende la innovación es inducida por la empresa cliente. }\end{array}$ \\
\hline TRADICIONAL & $\begin{array}{l}\text { La característica principal de las tradicionales es que sus productos no cambian o } \\
\text { cambian muy poco, por ende no ofrecen al mercado innovaciones y solo se centran en } \\
\text { aspectos estéticos dictados por tendencias de moda o relacionados con características } \\
\text { accesorias de los productos o de su forma de suministro. }\end{array}$ \\
\hline OPORTUNISTA & $\begin{array}{l}\text { Los que identifican nichos de mercado sin explotar y que se mueven rápidamente para } \\
\text { aprovechar las oportunidades de negocio emergentes. }\end{array}$ \\
\hline
\end{tabular}

Fuente: Elaboración propia a partir de Freeman y Soete (1997:168) y Robledo (2013).

Esta clasificación permite identificar diferentes opciones que tiene una organización al establecer sus estrategias de innovación de una manera deliberada, en la cual se supone que todas las variables de competencia, mercados, capacidades organizacionales, incluso la Ciencia y la Tecnología están plenamente establecidas. Esta condición de las variables no es aplicable a países de economías emergentes, como Colombia, en donde "la industria no descuella por su desarrollo tecnológico ni su dinamismo innovador, indudablemente, es imperativa una revisión a fondo de las estrategias empleadas por las empresas nacionales para hacer frente al acelerado cambio técnico $y$, antes que sufrir pasivamente la rápida obsolescencia de productos y procesos, actuar proactiva y flexiblemente para explotar las oportunidades que conlleva" (Robledo, 2013:102). En el cuadro 2 se clasifican las estrategias de innovación de Freeman (1997) de acuerdo al nivel de desarrollo tecnológico de la empresa y el nivel de participación en el mercado, lo que permite dar claridad a la categorización de las posibilidades estratégicas de innovación que tiene una empresa en el entorno competitivo. 


\section{Cuadro 2}

\section{Clasificación de estrategias de Innovación}

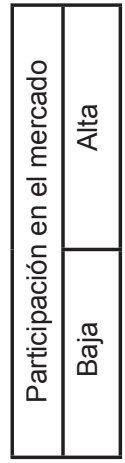

Desarrollo tecnológico de la empresa

\begin{tabular}{|c|c|}
\multicolumn{1}{c}{ Alto } & Bajo \\
\hline OFENSIVA & TRADICIONAL \\
\hline \multirow{3}{*}{ DEFENSIVA } & IMITATIVA \\
& DEPENDIENTE \\
& OPORTUNISTA \\
\hline
\end{tabular}

Fuente: Elaboración propia

Eiriz et al, (2013) proponen una clasificación de estrategias de innovación en función del tipo de innovación (productos y procesos) y su grado de novedad (incrementales y radicales), para ello se cruzan las decisiones que la empresa toma en el tipo y grado de innovación y se identifican cuatro tipos de innovación: desarrollo de productos, aprendizaje por la experiencia, descubrimiento y la restructuración (Gráfico 1).

\section{Gráfico 1}

Tipos de innovación según grado de novedad y tipo de innovación

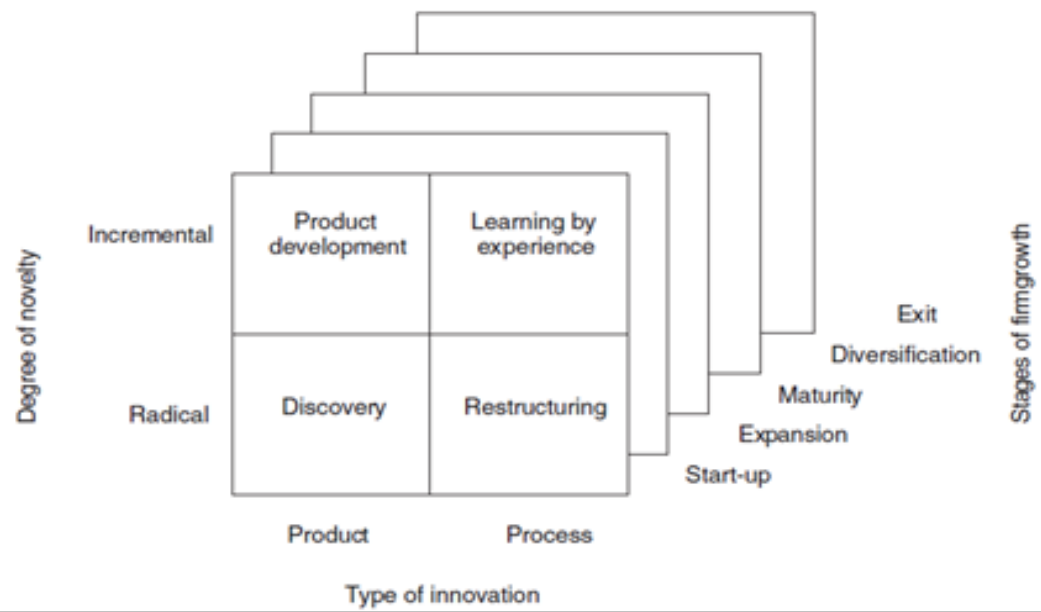

Fuente: Eiriz et al, (2013:104) 
Eiriz et al, (2013) hacen dos conclusiones importantes, en primer lugar que las decisiones en materia de innovación son los principales impulsores de las estrategias de innovación y tienen que ser objeto de reconocimiento con el tiempo para proporcionar evidencia empírica sólida. En segundo lugar se refieren a la importancia de los cambios en la estrategia de innovación de las empresas que se dan a través del tiempo y a través de las etapas de crecimiento de la misma.

Relación entre adquisición de TIC e innovación en Colombia: Análisis estadístico.

Para la recolección de datos se utilizó como fuente la segunda Encuesta de Desarrollo e Innovación Tecnológica (EDIT II) realizada por el Departamento Administrativo Nacional de Estadística -DANE- en Colombia, esta encuesta nacional de innovación busca, en general, aportar en la necesidad de conocer el estado actual del país con respecto a indicadores de innovación (Serna, Correa y Robledo, 2009) por tanto, constituyen una herramienta clave de análisis de la gestión tecnológica y la innovación en las empresas y sectores del país.

En Colombia se han llevado a cabo varias de estas encuestas. La Primera fue aplicada en el Establecimiento Industrial Colombiano y fue elaborada por el Departamento Nacional de Planeación (DNP) y Colciencias en el año 1996. La ejecución de esta encuesta permitió, por primera vez en Colombia, realizar una clasificación de las empresas del sector manufacturero de acuerdo a su nivel de innovación (Serna, Correa y Robledo, 2009).

La segunda EDIT se desarrolló a través de un acuerdo de cooperación técnica entre el Departamento Nacional de Planeación - DNP-, el Instituto Colombiano para el Desarrollo de la Ciencia y la Tecnología "Francisco José de Caldas" - COLCIENCIAS - y el Departamento Administrativo Nacional de Estadística - DANE- y se dirigió a la totalidad de las empresas industriales de acuerdo al directorio establecido para la Encuesta Anual Manufacturera (EAM), establecimientos industriales del país, que ocupen 10 o más personas o que obtengan una producción anual igual o superior a $\$ 65$ millones en el año de referencia; esto asegura que los resultados obtenidos tengan cobertura nacional y departamental.

Está estructurada en tres partes: la primera corresponde a la identificación de la empresa, ubicación, datos generales, tipo de organización, composición del capital social, número de establecimientos de la empresa, y la actividad económica según la Clasificación Industrial Internacional Uniforme (CIIU) de todas las actividades económicas, en su tercera Revisión, adaptada para Colombia; la segunda indaga sobre las actividades de desarrollo tecnológico y los objetivos de la innovación. La tercera parte indaga sobre las relaciones de las actividades de desarrollo e innovación tecnológica y el papel del Estado en cuanto al grado de conocimiento de los instrumentos de la política pública, y el financiamiento de actividades de desarrollo e innovación tecnológica; igualmente, evalúa la protección del conocimiento generado por las empresas a través de los diferentes componentes del sistema de propiedad intelectual y del sistema de acreditación y normalización técnica (DANE, 2004).

En esta encuesta las variables son muy específicas a la hora de identificar los procesos de innovación en las 
empresas. Por ejemplo, en el análisis del producto se utiliza una pregunta para cada tipo, de acuerdo con el concepto de novedad que plantea el Manual de Oslo (OCDE, 2005), es decir, innovaciones nuevas para la empresa, el mercado o el mundo entero. Por esta razón y debido a que el interés de este trabajo está centrado en la existencia de innovación y no en el análisis del alcance de la misma, se procedió a reducir las tres variables de clasificación de los tipos de novedades de productos en una sola variable que permita identificar si se ha hecho o no innovación en cada empresa encuestada.

En este sentido, el presente trabajo contribuye al análisis de la dinámica de la innovación en Colombia, mediante el estudio de una de las variables contempladas en la encuesta, la adquisición de tecnología. Finalmente se contrasta el resultado del análisis estadístico con la revisión de literatura, con el fin de determinar la pertinencia de la adquisición de TIC en el proceso de creación de la estrategia.

En la EDIT II se obtuvo información de 5.222 empresas de las 6.223 encuestadas, debido a que las demás no contaban con información acerca de estos procesos. Para determinar la adquisición de TIC y la innovación en las empresas colombianas se usaron las siguientes variables (Cuadro 3):

\section{Cuadro 3}

Variables de estudio

\begin{tabular}{|c|c|c|c|}
\hline Variable & Tipo de variable & Escala & Observaciones \\
\hline $\begin{array}{l}\text { Innovación } \\
\text { producto }\end{array}$ & $\begin{array}{l}\text { Nominal } \\
\text { Dicotómica }\end{array}$ & $\begin{array}{l}1=\text { Obtenido } \\
2=\text { No aplica }\end{array}$ & $\begin{array}{l}\text { Se realizó proceso de } \\
\text { reducción de variables: } \\
\mathrm{C} 2 \mathrm{~F} 1, \mathrm{C} 3 \mathrm{~F} 1 \text { y C4F1 y se } \\
\text { ajustó a dicotómica. }\end{array}$ \\
\hline
\end{tabular}

$\begin{array}{lll}\text { Adquisición de } & \begin{array}{l}\text { Nominal } \\ \text { TIC }\end{array} & 1=\text { Adquirió } \\ & \text { Dicotómica } & 2=\text { No adquirió }\end{array}$

Fuente: Elaboración propia

Después de identificar las variables de estudio, se sometieron a un análisis de tablas de contingencia y medidas de asociación que permitió conocer si hay relación y en qué grado. Aquí se prueba si la hipótesis sobre la adquisición de TIC está relacionada con la innovación mediante el rigor del análisis estadístico. En primer lugar se utilizó una prueba de independencia chi cuadrado para validar si hay dependencia entre las variables y en segundo lugar se utilizó el coeficiente de correlación Phi, que permite medir la intensidad de la relación entre las dos variables y así identificar el peso que tiene la adquisición de TIC, en las empresas para tener innovaciones de producto. 
Las variables establecidas se encuentran en la misma escala de medición, por ende, se utilizó un coeficiente de correlación que verifique la intensidad de la relación entre estas variables dicotómicas. Cabe mencionar que la variable innovación de producto en la segunda encuesta es originalmente politómica, pero debido a que no se presenta ninguna respuesta con innovaciones de proceso, se tomó como dicotómica. Además se realizó un proceso de reducción de variables que consistió en elegir si la empresa ha obtenido algún tipo de innovación de producto de cualquiera de los tres presentados por la encuesta "a nivel empresarial, del mercado o del mundo entero".

Para el análisis de la relación existente entre las dos variables se usó una tabla de contingencia que se utiliza según Canavos (1988:370) "cuando es necesario determinar si existe una relación entre dos rasgos diferentes en los que se ha clasificado una población y cada rasgo se encuentra dividido en categorías donde por lo menos dos son exhaustivas y mutuamente excluyentes"

Para determinar si existe una relación de dependencia entre las variables obtenidas, se utilizó la prueba chi-cuadrada, que permitió identificar que las dos clasificaciones son independientes, esto es, bajo la hipótesis nula de independencia se busca identificar si existe una diferencia suficiente entre las frecuencias observadas y las esperadas, tal que la hipótesis nula se rechace (Canavos, 1988:371).

Finalmente el coeficiente para medir la intensidad de la relación entre las variables según Torres y Mejias (2005) es el coeficiente de correlación $\mathrm{PHI}$, el cual se utiliza cuando es necesario identificar la intensidad de la relación entre dos variables dicotómicas, el símbolo asignado es $\Phi$. El coeficiente fluctúa entre cero y uno. Según se acerca a cero, más baja es la asociación entre las variables. Si es cero, las variables no están afectadas o relacionadas entre sí, estableciendo que las variables serían independientes. Si el coeficiente es igual a uno lo que se puede sugerir es que las dos variables están relacionadas o asociadas, es decir, las variables son dependientes.

En la tabla 1 se ilustran las frecuencias observadas y esperadas en la tabla de contingencia:

\section{Tabla 1}

Tablas de contingencia

Valores Observados

Adquisición de TIC

Innovación de producto

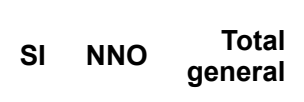

Valores Esperados

Adquisición de TIC

Innovación

de producto

SI

NNO

Total

general

\begin{tabular}{llll}
\hline Obtenida & 235.3142 & 495.69 & 731 \\
No aplica & 1445.686 & 3045.3 & 4491 \\
Total general & 1681 & 3541 & 5222 \\
\hline
\end{tabular}

Fuente: Elaboración propia 
Para esta tabla de contingencia se procedió a establecer la dependencia entre las variables a través de la prueba de independencia chi cuadrado, que se planteó de la siguiente forma:

$H_{0}$ : Hay independencia entre el uso de TIC e innovación de producto

$H_{1}$ : Hay dependencia entre las variables analizadas

\section{$\propto=0.05$}

A partir de la distribución de frecuencias en la tabla de contingencia se calcula el $X^{2}$ (chi cuadrado) que fue de 4.80 en comparación con el $X^{2}$ 0.95, $(r-1)(k-1)$ que es de 3.84. A partir de ahí, puede concluirse que hay evidencia estadística para rechazar la hipótesis nula, con lo cual se demuestra que hay dependencia entre el uso de las TIC y la innovación de producto.

Para medir la intensidad de la relación entre las variables se utilizó el coeficiente de correlación PHI, que presento los siguientes resultados.

$\phi$ Obtenido $=0,02975$

$\phi$ Máximo $=0,58496$

$\phi$ Corregido $=0,05086$

Este coeficiente muestra que la intensidad de la relación entre la adquisición de TIC y la innovación de producto es baja (0.05086), es decir, el que una empresa adquiera TIC puede traer ventajas para la innovación, pero no necesariamente se convertirá en un factor determinante en el proceso de innovación de productos.

En el análisis de la relación entre el uso de TIC y la innovación se encontró evidencia estadística que muestra que hay dependencia entre las variables, es decir el uso de TIC en las empresas sugiere la existencia de innovaciones en la misma, aunque no se evidencia una gran intensidad en la relación de dependencia, factor atribuible, por ejemplo, a que las empresas no utilizan este tipo de tecnologías en el área productiva, sino en las labores corrientes de oficina.

La utilización de la encuesta presentó vacíos a la hora de analizar el uso de tecnologías, dado que solo toma en cuenta la última tecnología adquirida y no el inventario tecnológico de la empresa lo que constituye una debilidad a la hora de analizar el potencial real de tecnología en la empresa. La imposibilidad de analizar por sectores los datos de la segunda encuesta, también limitó el estudio del impacto que tiene esta tecnología en la innovación de las empresas en los diferentes sectores, porque a pesar de que se encuentra relación entre las dos variables es posible que cada sector se comporte de una manera diferente en la intensidad de la relación.

\section{Conclusiones}

La adquisición de TIC en las empresas tiene relación con la innovación sin evidenciar alta asociación entre las variables, lo que indica que en las empresas colombianas la adquisición de este tipo de tecnología no es parte activa de la estrategia de innovación o su uso se ha orientado hacia actividades que no generan ventaja competitiva en la producción de bienes, como pueden ser las administrativas o de oficina.

La gestión de recursos en la organización juega un papel importante, pues la adquisición de TIC, por sí sola no representa una ventaja, por ende es necesario adaptar la tecnología al desarrollo productivo mediante la formulación de estrategias que busquen desarrollar ventajas a partir de esta, y producir así resultados en innovación para la empresa. 
La gestión de la tecnología y la innovación en las empresas a través de procesos de planeación tecnológica e implementación de políticas y estrategias de desarrollo tecnológico, juega un papel fundamental en el aprovechamiento que se tiene de la alta tecnología en las empresas, pues se evidencian falencias en este aspecto en las empresas colombianas, manifestados en este caso, en un bajo impacto de las TIC.

La innovación es estratégica para las organizaciones porque le permite obtenerventajas competitivas sostenibles en el tiempo, por ende es necesario comprender su proceso de generación; cuáles son sus principales variables, como se forman estas innovaciones y que capacidades se requiere para ello. Todo esto se puede representar en estrategias que la empresa define para poder alcanzar las innovaciones, es decir, las decisiones tomadas en materia del uso de tecnología, a través de las diferentes etapas de su desarrollo evolutivo. Se requiere de una mirada holística al proceso de innovación a partir del componente tecnológico, y la formación de estrategias al integrar capacidades y recursos se convierte en el mejor escenario para ello.

Por tanto, es necesario tener en cuenta que la implementación de estrategias para orientar el uso de este tipo de tecnología al desarrollo de innovaciones por medio de una adecuada gestión de recursos tecnológicos; comenzar por conocer el inventario de tecnologías de la organización e identificar debilidades y fortalezas y los planes de acción para convertirlas en oportunidades.

Es necesario que las empresas dispongan de apoyos metodológicos para la innovación, integrando adecuadamente su componente tecnológico que posean para alcanzar el desarrollo de verdaderas capacidades de innovación, los cuales se materializan en estrategias más adecuadas.

Los datos suministrados por las encuestas de innovación presentan limitaciones para un análisis más riguroso, debido a la imposibilidad de tener una ventana de tiempo más amplia en la recolección de los mismos y por ende trabajar con unas variables más desarrolladas. En el caso de la variable de adquisición de tecnologías, sería más apropiado tener datos acerca del tipo de tecnología manejada por la empresa y medir factores como el uso o la aplicación, que serían más explicativos para el proceso de innovación.

Como estudios futuros se plantea la necesidad de analizar a fondo las implicaciones de la adquisición y uso de las altas tecnologías en el desarrollo de la innovación, con el fin de caracterizar este proceso y proponer metodologías acordes a las necesidades de las empresas colombianas. En cuanto al uso de las encuestas de innovación, se requiere mejorar la presentación de la información para hacer estudios más profundos y con mayor rigurosidad.

\section{Referencias bibliográficas}

Aït-El-Hadj, Smail (1990), Gestión de La Tecnología. La Empresa Frente a La Mutación Tecnológica. Estados Unidos: Ediciones Gestión 3000.

Barney, Jay (1991), "Firm Resources and Sustained Competitive Advantage." Journal of Management 17(1): 99120.

Bell, Martin, y Keith Pavitt (1995), "The Development of Technological Capabilities." In Trade, Technology and International Competitiveness, Washington: The World Bank, 69101. 
Burgelman, Robert, Maidique, Modesto and Wheelwright Steven (2001), Strategic Management of Technology and Innovation. ed. Translated in Chinese in 2004. New York: McGraw-Hill/Irwin.

Canavos, George (1988), Probabilidad y estadística: análisis y métodos. Mc Graw Hill

Christensen, Jens Frøslev (1995), "Asset Profiles for Technological Innovation." Research Policy 24(5): 727-45.

Cortés, Elkin Alonso (2004), La Gestión Y Transferencia Tecnológica Cruce de Caminos Entre UniversidadEmpresa. Universidad Nacional de Colombia - Sede Medellín.

Cory, Joseph (1999), Una Arquitectura de Los Negocios Para La Gestión Tecnológica. Colombia: McGrawHill.

DANE (2004), Innovación Y Desarrollo Tecnológico. En La Industria Manufacturera. Colombia 2003 2004.

Drejer, Anders (1996), "Frameworks for the Management of Technology: Tov I Rards a Contingent Approach." Technology Analysis \& Strategic Management 8(1): 13.

Eiriz, Vasco, Ana Faria, and Natália Barbosa (2013), "Firm Growth and Innovation: Towards a Typology of Innovation Strategy." Innovation: Management, Policy \& Practice 15(1): 97-111. http:// pubs.e-contentmanagement.com/doi/ abs/10.5172/impp.2013.15.1.97.

Eisenhardt, Kathleen and Martin, Jeffrey. (2000), "Dynamic Capabilities: What Are They?" Strategic Management Journal 21(10-11): 1105-21. http:// www.jstor.org/stable/3094429.

Escorsa, Pere, y Pasola Valls (1998), Tecnología e Innovación En La Empresa. Ediciones UPC.

Fernández, Esteban (2005), Estrategia de Innovación. Thomson. Madrid

Freeman, Chris (1982), The Economics of Industrial Innovation. Segunda. Londres: Francis Pinter.
Freeman, Chris, y Luc Soete (1997), The Economics of Industrial Innovation. Tercera. Psychology Press.

Galbraith, John Kenneth. (1967), The new state. Houghton Mifflin. Boston, USA

Gallego, José Benjamín (2005), "Fundamentos de La Gestión Tecnológica E Innovación." Tecnológicas ITM Diciembre(15).

Hamel, Gary (1998), "Opinion Strategy Innovation and the Quest for Value." Sloan Management Review: 7-14.

Lall, Sanjaya (1992), "Technological Capabilities and Industrialization." World Development 20(2): 165-86.

Londoño, John (2010), “Estudio de La Relación Entre Alta Tecnología E Innovación En Colombia." Universidad Nacional de Colombia Sede Medellí

Malaver, Florentino (2000), Investigación En Gestión Empresarial: Proceso Naciente?: Colombia, 1965-1998. Bogotá: Corporación Calidad.

Markides, Constantinos (1997), "Strategic Innovation." Sloan Management Review: 9-23.

Nelson, Richard R. (1991), "Why Do Firms Differ, and How Does It Matter?" Strategic Management Journal 12: 61-74. http://doi.wiley.com/10.1002/ smj.4250121006.

O'Regan, Nicholas, y Martin A. Sims (2008), "Identifying High Technology Small Firms: A Sectoral Analysis." Technovation 28(7): 408-23.

OCDE (2005), Manual de Oslo: Proposed Guidelines for Collecting and Interpreting Innovation Data. Tercera. Paris.

Olaya, Alejandro (2008), "Economía de La Innovación Y Del Cambio Tecnológico: Una Aproximación Teórica Desde El Pensamiento Schumpeteriano." Revista Ciencias Estratégicas. 16(20): 237-46.

Penrose, Edith (1959). The Theory of the Growth of the Firm. ed. Oxford: Basil 
Blackwell. New York: John Wiley \& Sons.

Porter, Michael (1980), Competitive Strategies: Techniques for Analyzing Industries and Competitors. New York: MacMillan.

Prahalad, Coimbatore Krishnarao, and Hamel, Gary. (1990), "The Core Competence of the Corporation" eds. Dietger Hahn and Bernard Taylor. Harvard Business Review 68(3): 79-91. http://www.springerlink.com/ index/v1774282g031q747.pdf.

Robledo, Jorge (2013), Introducción a la Gestión de la Tecnología y La Innovación. Medellín: Universidad Nacional de Colombia - Sede Medellín (Facultad de Minas).

Robledo, Jorge, Fredy Gómez, y Juan Felipe Restrepo (2009), "Relación Entre Capacidades de Innovación Tecnológica Y El Desempeño Empresarial $Y$ Sectorial." In Encuestas, Datos $Y$ Descubrimiento de Conocimiento Sobre La Innovación En Colombia, 71-100.

Rosenberg, Nathan. (1982), Inside the black box: Technology and economics. Cambridge, UK: Cambridge University Press.

Sanches, Paula Luciana Bruschi y André Gustavo Carvalho Machado (2014), "Estratégias de Inovação Sob a Perspectiva Da Resourced-Based View: Análise $E$ Evidências $E m$ Empresas de Base Tecnológica." Gestão \& Produção 21(1): 125-41. http://www.scopus.com/ inward/record.url? eid=2-s2.0 -
84899485152\& partnerID=tZOtx3y1 (November 27, 2014).

Serna, Sandra Carolina; Correa, Juan Carlos and Robledo, Jorge. (2009), Análisis Comparativo entre Árboles de Regresión y Clasificación (CART) y Regresión Logística: Aplicación a la Caracterización de Empresas Innovadoras Colombianas. En: Encuestas, Datos $Y$ Descubrimiento De Conocimiento Sobre La Innovación En Colombia. Vol. 1, págs.: 235 - 264, Ed. Fundación Cultural Javeriana De Artes Gráficas Javergraf

Snarch, Alejandro (1998), "La Innovación: Estrategia Del Desarrollo Empresarial."

Teece, David; Pisano, Gary and Shuen, Amy. 1997. "Dynamic Capabilities and Strategic Management" eds. Giovanni Dosi, Richard R Nelson, and Sidney G Winter. Strategic Management Journal 18(7): 509-33. http://doi. wiley.com/10.1002/(SICI)10970266 (199708)18:7<509::AID SMJ882>3.0.CO;2-Z.

Torres Degró, Arnaldo and Mejías Afanador, Evelyn. (2005). Estadística Descriptiva en el Campo Social Puertorriqueño. Primera edición, Antillana College Press, Mayagüez, PR

Tidd, Joe (2000), "The Competence Cycle: Translating Knowledge into New Processes, Products and Services." In Knowledge Management to Strategic Competencies: Measures of Technological. Market \& Organisational Innovation, Londres: Imperial College Press. 